\title{
Denken und Fühlen als Mittel magischen Handelns
}

Research on folk beliefs and magic has thus far focused mainly on the description of supernatural beings and ritual sites; and the forms of folk belief lacking distinct verbal expressions have generally been ignored. These kinds of folk beliefs usually involve mental processes of which the subject is only partly aware, but nevertheless considers the thoughts and feelings meaningful.

Belief in the evil eye and the emotions which bring it about (ill will or envy) is both ancient and universal. This article begins with an examination of the magic practised by peasants and then proceeds to describe the thought processes of present-day city dwellers. Examples of the latter are taken from material gathered by the Department of Folklore at the University of Helsinki.

The childish inclination to think magically (i. e., the child imagines he can influence the world with his thoughts) is considered to be innate by some psychologists. Obsessive-compulsive illusions contain unconscious attempts to repress/repel oppressive notions; these illusions are made up of individually invented rituals. By indulging in various fantasies a person tries to mentally influence events and even the course of his life.

People do not have clear concepts of how emotions and fantasies could possibly affect their good luck (are these questions of the laws of nature, fate, a supernatural being/power?) Many of the beliefs that people use to bargain with the supernatural are selfcontradictory.

Der Mensch lebt nicht in der äußeren Realität inmitten von Salzen und Säuren, er lebt vielmehr in der warmen, träumerischen Kammer seines Gehirns, wo die Fenster bunt und die 
Wände voller Märchen sind, so hat es R. L. Stevenson ausgedrückt. ${ }^{1}$ Wir haben hier einen Hinweis auf die Fähigkeit des Menschen, die Welt nach seinem Belieben zu gestalten, seine innere Kammer gleichsam auszustatten mit Glaubensvorstellungen und Gedanken, die dem kalten Licht der äußeren Realität nicht standhalten.

Die Magie ist definiert worden als eine Reihe von unterschiedlichen Verfahren, durch die der Mensch sein Glück zu manipulieren versucht. Indem er das tut, ist er nicht abhängig vom Supranormalen, vom Übernatürlichen, wie der religiös eingestellte Mensch, sondern er nutzt dies auf unterschiedliche Weise aus, und die Folge ist automatisch das Ergebnis, wenn die Maßnahme als Ursache durchgeführt ist. ${ }^{2}$ In der Praxis sind jedoch magische und religiöse Verfahren nahtlos miteinander verbunden.

Zum magischen Verhalten gehören allerdings auch die Gedanken und Gefühlssituationen, die dem Handeln zugrunde liegen. Magie und Aberglauben machen eine breite Skala menschlichen Verhaltens aus, ebenso gut Emotionen und kaum bewußt werdende Gedankengänge wie in Worte gefaßtes Wissen und direktes, manipulierendes Handeln. Zwischen Glaubensvorstellung, Stimmung resp. Gefühlslage und Tätigkeit läßt sich schwer unterscheiden, denn das menschliche Handeln beruht auf inneren Vorstellungen und Emotionen. Eine Form der Magie besteht denn auch darin, daß man die Emotionen und Gedanken als real wirkend erlebt.

Magisches Denken läßt sich charakterisieren als eine Reihe von halbbewußten Prozessen, die im Menschen vorgehen, als unterbewußte Wirkungsmechanismen, wo nicht nachgedacht wird über die Beschaffenheit des Vorgangs zwischen Ursache und Wirkung. $\mathrm{Zu}$ definieren sind die strittigen Begriffe Sinn, Gefühl, Bewußtsein und vor allem Unterbewußtsein, dem in der psychoanalytischen Theorie eine zentrale Stellung zukommt. Zum Unterbewußtsein gehört jenes psychische Material, das aus dem Bewußt- 
sein verdrängt ist. Es ist dies gleichsam eine separate Denkweise, die um die Beherrschung des Verhaltens konkurriert.

Der Ausdruck „Unbewußtes“ läßt sich auch im weiteren Sinne als Bezeichnung für jenen Teil des Bewußtseins verwenden, dem die für das Bewußtsein typische Klarheit und Anschaulichkeit fehlen. Dazu gehören u. a. die unbestimmten Randgebiete des Bewußtseins, Empfindungen und Wahrnehmungen, die keine genaue Gestalt annehmen, passiv und aktiv vergessene Vorstellungen, sprachlich schwer auszudrückende Bewußtseinsinhalte usw. Für das Unbewußte gelten verschiedene Grade der Klarheit und der Differenzierung, doch sind es keine selbständig, auf verschiedene Weise „denkenden“ Teile, wie der finnische Psychologe Lauri Rauhala es ausdrückt. ${ }^{3}$ Wenn das magische Denken oft als unbewußt charakterisiert wird, so gehören dazu gerade jene Vorstellungen und Emotionen, die keine klare Gestalt angenommen haben und die auch nicht immer einen sprachlichen Ausdruck erhalten.

Das Sammeln entsprechenden Materials ist jedoch schwierig, da die Anschauungen nicht immer verbalisiert werden können. Über die Auswirkungen von Aberglauben und Zauber wird in der Regel nicht gern gesprochen in Gemeinschaften, wo man an sie glaubt. I. K. Inha berichtet in seiner Reisebeschreibung aus Weißmeerkarelien (1911), man spreche wenig von abergläubischen Vorstellungen, Zauber und Vorzeichen, glaube aber allgemein daran. „In aller Stille beherrschen sie das Leben des Volkes und ihre Kenntnis würde dem Wanderer vielerlei Ängste, Streitigkeiten oder sonstige Gemütsbewegungen erklären, auf die er aufmerksam wurde in den Hütten oder auch draußen, die ihm aber ein Rätsel geblieben sind." 4 Die Beschreibung der im Innern des Menschen entstehenden Gefühle und der halbbewußten Gedankenprozesse kann als wesentlicher Teil jenes Forschungsprozesses angesehen werden, dessen Ergebnis die Aufzeigung der kultureigenen Schematisierungen ist. 
Bei der Erforschung der Religion der finnisch-ugrischen Völker hat man sich meistens für die konkreten und sichtbaren Formen des Volksglaubens interessiert: beispielsweise die Todesrituale, die Opferhaine oder die kosmogonischen Auffassungen, die von jeher als würdige Forschungsobjekte galten. Die magischen Denkschemata könnte man in grober Vereinfachung vergleichen mit der untersten Abfallschicht, dem Unterholz im menschlichen Gemüt, in der menschlichen Seele: die hier entstehenden Vorstellungen - ob angsterfüllt oder hoffnungsvoll steuern jedenfalls das Verhalten.

Im folgenden soll die Bedeutung der Gefühle und Emotionen in der alten Agrarüberlieferung aufgrund jenes finnischen Materials geschildert werden, das in den Sammlungen des Volkskunde-Archivs der Finnischen Literaturgesellschaft aufbewahrt wird. Die Belege für die Gegenwart stammen aus den Sammlungen des Instituts für Folkloristik an der Universität Helsinki und betreffen die achtziger Jahre. Sie basieren häufig auf Interviews, die im Zusammenhang mit meiner Vorlesungsveranstaltung über modernen Volksglauben durchgeführt wurden. Der moderne Volksglauben setzt sich aus heterogenen Elementen zusammen, die im einzelnen schwierig zu finden, aufzuzeigen und zu definieren sind. Magische Überlieferung kann man paradoxalerweise eigentlich erst dann sammeln, wenn man ihre Beschaffenheit wenigstens in einem gewissen Ausmaß kennt. ${ }^{5}$

Zur Bedeutung der Gefühle in der alten Magie

Die Bedeutung der Gefühle und Gedanken kommt vor allem in der uralten, weltweit bekannten Vorstellung vom bösen Blick zum Ausdruck. Die Ursache für den Verlust des Glücks liegt nicht in einer Tätigkeit, dem Blicken, sondern in der Gefühlslage an sich, einer starken Emotion, dem bloßen bösen Gedanken. 
Noch im 18. Jahrhundert konnte in Skandinavien jemand vor Gericht verklagt werden, von dem man vermutete, er habe etwas Böses gedacht, während er in der Stube auf der Bank saß.

Die Wirkung des bösen Blicks beschränkt sich also nicht unbedingt auf das den Menschen symbolisierende Auge oder den Blick. Der böse Blick konnte auch aufgefaßt werden als eine Kraft oder Energie, deren Einfluß völlig unabhängig ist vom Willen, die der Mensch nicht selbst beherrschte; so jemand wagte es zum Beispiel nicht, auch nur die eigenen Haustiere anzuschauen, da sie sonst gegen seinen Willen krank geworden wären. ${ }^{6}$

Feindseligkeit enthält schon an sich als Gefühlszustand eine geheimnisvolle, schädigende Kraft. Gebrochen werden kann diese Kraft auf mancherlei Weise, indem man z. B. auf Haß mit $\mathrm{Ha} \beta$ antwortet. Durch grobe, schändliche Worte kann man den Sinn des Verneiders so durcheinanderbringen, daß er seine Fähigkeit, durch den bösen Blick Schaden anzurichten, einbüßt. Magische Vorsorge war auch in ganz normaler Gastfreundschaft enthalten: Es war eine vorbeugende Maßnahme, dem Gast im Haus einen Schnaps anzubieten, denn einerseits verliert das Verneiden dadurch seine Kraft, andererseits freut sich der Betreffende über den Trank und verschont z. B. das in der Wiege schlafende Kind vor seinem bösen Blick. ${ }^{7}$

Die eigentliche böse Wirkung rührt hier von den Gedanken des Menschen her; der Blick und die Worte, Fluch, Verwünschung oder Wunsch sind nur Verstärker der Wirkung und deutliche Ausdrücke. Ein solcher Glaube war selbstverständlich in einer face to face-Gemeinschaft, wo man die Ereignisse des Lebens auch sonst durch enge menschliche Kontakte erklärte.

Ein gutes Beispiel dafür, wie die Emotionen als Kraft verstanden werden, sind die im alten Fischfangzauber enthaltenen Verbote, die den Fischer vor einer zornigen, streitsüchtigen Gemütslage warnen. Das ostseefinnische Sprichwort kateus vie kalat vedestä ('Der Neid nimmt die Fische aus dem Wasser') besaß 
eine große Beliebtheit und Verbreitung; charakteristisch und eindrucksvoll bei der sprachlichen Ausformulierung ist die Alliteration. Heute ist es nur noch eine bildliche Ausdrucksweise, seinerzeit aber glaubte man, die Fischgründe würden verdorben, falls die Fischer bei ihrer Arbeit streiten oder die Fische, zumindest bestimmte Arten, würden verschwinden. Durch Fluchen, Zanken, überhaupt ein zorniges Verhalten auf dem Wasser wird der gleiche Schaden angerichtet; schon allein eine streitsüchtige Stimmung kann das Fischglück oder das Fischgewässer verderben.

Eine ausgeglichene, ruhige Gemütsverfassung war für den Fischer also von Vorteil. Wenn Fischfanggeräte hergestellt werden, darf darüber nicht argumentiert werden; die Ausbeute an Fischen darf nicht für zu gering oder für zu reichhaltig betrachtet werden, man darf keine streitsüchtigen Gedanken haben, nicht streiten und nicht fluchen. Eine harmonische, ausgeglichene Stimmung garantiert dem Fischer das beste Ergebnis. ${ }^{8}$

Ein altes Beispiel für die Regulierung der Emotionen bilden auch die mit den Klageliedern zusammenhängenden Auffassungen. Wenn die karelische Braut zum Weinen gebracht wurde, indem sie Klagelieder sang also, so war das - nach der karelischen Forscherin Unelma Konkka - eine der Vorsichtsmaßnahmen, durch die sie vor dem Groll der supranormalen Kräfte geschützt wurde. Die Braut mußte weinen, um sich das Mitgefühl der Verstorbenen zu sichern. ${ }^{9}$ In dieser Auffassung ist vermutlich der ursprünglichste Sinn erhalten; später hat man dann gesagt, wenn die Braut auf der Hochzeit weine, dann brauche sie nicht im Haus ihres Mannes zu weinen. Eine zu große Sicherheit und eine voreilige Freude sind von Übel. In der karelischen Überlieferung kommt die alte Auffassung zum Ausdruck, daß die übernatürlichen Kräfte - in diesem Fall die Toten - sich rächen können an jemandem, der sich zu sehr freut; auch die alten Griechen fürchteten die Rache der Götter, die Nemesis, denn auch die Götter waren nicht glücklich. ${ }^{10}$ 
Vorstellungen von der Wirkung der Emotionen finden sich im alten Volksglauben, obwohl sie nicht eigens aufgezeichnet worden sind. Vertreten sie nun ein Denken, das für die Menschen überall in der Welt typisch ist, oder lernt man diese Auffassungen durch Vermittlung der eigenen Kultur? Ein als magisch zu bezeichnendes Denken begegnet häufig bei Kindern sowie bei bestimmten psychischen Krankheiten; durch diese Bereiche läßt sich die Auffassung untermauern, wonach magisches Denken in unterschiedlichen Gegenden ohne kulturelle Verbreitung entsteht.

Kindermagie

Kindermagie ist teilweise sozial überlieferte Tradition, teilweise besteht sie aus individuell entwickelten, geheimen Ritualen aufgrund der Ideen, die die jeweilige Kultur vermittelt. Im Denken der Kinder sind viele Beispiele für Vorstellungen enthalten, die von der Omnipotenz der Gedanken zeugen.

Manche Psychologen halten die kindliche Neigung zu magischen Gedankengängen für angeboren. Jean Piaget, bekannt für seine Untersuchungen über die Entwicklung des Kindes, spricht von animistischem und magischem Denken als allgemeinmenschlichen Entwicklungsstadien. Wenn das Kind merkt, daß Sonne und Mond ihm folgen, kann es dies entweder animistisch (sie verfolgen mich) oder magisch erklären (ich kann sie bewegen). ${ }^{11}$

Die übliche Magie in der Kindheit besteht darin, Rituale zu entwickeln, die Sicherheit schaffen. In der Regel erfahren die Eltern nichts davon, und das Kind baut sie in seinem eigenen Sinn. Sie gelten entweder dem Abwenden eines drohenden Übels oder dem Erreichen einer bestimmten Sache. Ein kleines Mädchen sitzt am Fenster und beschließt, bis hundert zu zählen; in dieser Zeit müßte die Mutter nach Hause kommen. 
Allgemein ist auch das Wünschen als Magie bei Kindern. Man darf sich etwas wünschen (und dabei glauben, daß der Wunsch in Erfüllung geht), wenn man dasselbe Wort gleichzeitig mit einem anderen ausspricht, wenn eine ausgegangene Wimper beim ersten Pusten in die Luft fliegt, wenn man ein vierblättriges Kleeblatt findet usw. Die Situation des Wünschens wirkt direkt wie aus dem Märchen. Es ist allerdings nicht die gute Fee, die einen etwas wünschen läßt, sondern der Mensch bemerkt es selbst oder entwickelt die Situation mit Hilfe eines besonderen Ritus (indem er z. B. die Nummernschilder von hundert Autos zählt). Der Wunsch wird nicht laut ausgesprochen und man denkt auch nicht darüber nach, wer den im Stillen geäußerten Wunsch hört und erhört.

Schon dadurch, daß man seine Aufmerksamkeit auf etwas richtet, kann dieses Objekt bereits magische Bedeutung erlangen. Vor allem Kinder vermeiden es, auf die Grenzlinien der Straßenpflasterung zu treten; für sie ist diese Vorsicht gleichzeitig eine Form des Zeitvertreibs. Man sieht sich vor, auf die Linien zu treten, wenn man sonst an nichts besonderes $\mathrm{zu}$ denken hat. Vielleicht fürchtet sich der oder die Betreffende, daß sich die Erde spaltet oder daß einen die schwarze Tiefe verschlingt (oder etwas ähnliches), aber Unglück bringt das Betreten der Linien nur dann, wenn man - wie in der Magie überhaupt - besonders an die Angelegenheit denkt. Ein Kind, das es eilig zur Schule hat, achtet nicht auf die Pflasterung der Straße.

Das Kindsein ist eine problematische soziale Situation, die auf ihre Weise die Erhaltung des magischen Denkens fördert. Die Kinder können sich schwer ein Bild machen von dem wirklichen Verhältnis zwischen Ursache und Wirkung, denn die Welt wird ihnen anders dargestellt als den Erwachsenen. Von einem kleinen Kind erwartet man, daß es an den Weihnachtsmann glaubt und sich anstrengt, artig zu sein; man liest ihm Märchen und Comics vor, in denen Tierfiguren wundersame Dinge erleben, und wenn 
ein Kind zu seinem Spielzeug spricht, verhalten sich die Erwachsenen oft so, als wäre das Spielzeug wirklich lebendig.

Die absichtliche Magisierung der Welt der Kinder kann damit verteidigt werden, daß sich ihre Phantasie entwickelt, doch wird durch eine solche Einstellung gleichzeitig der Rangunterschied zwischen dem Kind und dem Erwachsenen erhöht. Nur der Erwachsene glaubt zu verstehen, was wirklich existiert. Sehr selten wagen Kinder Erwachsenen gegenüber offen von den sie bedrückenden Ängsten zu sprechen.

Magische Vorstellungen werden in der Kindheit teils durch die Gruppe der Altersgenossen, teils durch die Eltern und die sonstige Umwelt beeinflußt und übernommen. Hinter individuell wirkenden Ritualen stehen eine bestimmte gemeinsame kulturelle Basis und durch die Kultur gebotene Bedeutungen, die das Individuum auf die Praxis anwendet. Der finnische Philosoph Eino Kaila hat darauf hingewiesen, daß etwas bewußt für wahr zu halten bereits eine sehr spezifizierte Verhaltensweise darstellt, die eine hohe geistige Entwicklung voraussetzt; auf den niedrigeren Stufen stehen sich das Geglaubte und das Vorgestellte viel näher. ${ }^{12}$ Auch wenn das Kind nicht mehr wie in der Psychologie vom Anfang des Jahrhunderts als primitiver Erwachsener gilt, lassen sich doch in der Entwicklung der Begriffe verschiedene Phasen unterscheiden.

Zwangsvorstellungen

Die Psychiatrie hat sich besonders mit neurotischen Patienten beschäftigt, deren späteres Leben durch eine magische Bedrohtheit gekennzeichnet ist. Die sog. zwangsneurotischen Symptome bestehen aus Zwangsvorstellungen oder Obsessionen und Zwangshandlungen. Die Gedanken beherrschen den Menschen anstatt daß der Mensch seinen Gedanken die Richtung und die Grenzen 
weist. Die Gedanken scheinen aus dem Innersten des Menschen zu kommen, ohne feststellbare Muster in der Umwelt.

Viele Menschen haben leichte zwangsneurotische Züge (man überprüft, ob die Tür abgeschlossen ist, obwohl man weiß, daß das der Fall ist; man versucht, Vorzeichen zunichte zu machen, z. B. indem man nach einer schwarzen Katze ausspuckt). Die Erscheinungen schwanken: es gibt harmlose, als normal zu bezeichnende Merkmale bis hin zu Symptomen, die den Betreffenden invalidisieren. Die Zwangsvorstellungen sind häufig bedrückende Gedanken über Handlungen, die gegen die Moralvorstellungen verstoßen; andererseits beinhalten sie ein Zählen oder Aufzählen, das in Gedanken geschieht. ${ }^{13}$ In den Zwangsvorstellungen ist eine magisch geartete Idee darüber enthalten, wie man mit ihrer Hilfe ein als unbestimmt empfundenes Übel umgeht.

Auch das Zählen, Aufzählen und zwangshafte Rituale, wie sie für Kinder charakteristisch sind, all dies erhält mitunter deutlich neurotische Züge. Hinter diesem Verhalten steht - wie übrigens auch bei den Erwachsenen - ein Gefühl der Bedrückung, deren eigentliche Ursache nicht deutlich erfaßt wird. Die Kinder können zum Beispiel davor Angst haben, daß die Eltern sterben oder auch, daß sich der Betreffende vor seinen Freunden unglaublich blamiert; der Grund der Bedrängnis wird allerdings oft gar nicht erfaßt, was wiederum typisch ist für dieses Gefühl.

In der Forschung ist darauf aufmerksam gemacht worden, daß das Über-Ich des Zwangsneurotikers äußerst streng ist. Seine Rituale sind durch das Gewissen erzwungene Versuche, das Übel, das seine Erfüllung verlangt, auf magische Weise fortzuzaubern oder zunichte zu machen. Die Grenze zwischen den normalen Denkvorgängen des Menschen und zwischen entsprechenden Störungen ist nicht immer deutlich; bei der Beurteilung dieser Frage muß auch die umgebende Kultur mitberücksichtigt werden. $\mathrm{Zu}$ den Störungen der Geistestätigkeit gehören auch die Wahnvorstellungen, in denen die Wirklichkeit mit den gefühlsmäßigen 
Bedürfnissen der Persönlichkeit gleichgesetzt wird. Wahnvorstellungen können definiert werden als falsche Glaubensvorstellungen, durch die die Fähigkeit des Menschen, Brücken zu bauen zwischen seinen inneren Phantasievorstellungen und der Wirklichkeit ins Wanken geraten ist.

Bedingungen als Vorzeichen

Das in unserer Zeit gesammelte Material von Glaubensvorstellungen enthält sowohl Beispiele für Stimmungs- bzw. Geisteszustandsmagie als auch für kleine magische Rituale, die man in Gedanken durchführt.

Mitunter setzt man sich Bedingungen als Vorzeichen. Wenn man die Bedingung erfüllen kann, z. B. etwas rechtzeitig schafft, so hat man Erfolg in der Angelegenheit, die gerade Gegenstand des Interesses ist. Ein Vorzeichen befragt man in der Regel allein und spricht auch nicht zu anderen davon. Man akzeptiert oder verwirft das Vorzeichen, und oft vergißt man die ganze Angelegenheit, wenn man sich nicht mehr bedrängt fühlt. Man kann zum Beispiel beschließen, daß man mindestens drei Ampeln bei grün erreichen muß, sonst bringt der Tag Unglück.

„Ich mußte z. B. auf den Schatten des folgenden Telefonmastes treten, ehe ein bestimmtes auf der Straße fahrendes Auto um die Ecke biegt oder ich mußte vom Sandweg auf Asphalt kommen, ehe das Flugzeug am Himmel eine bestimmte katzenförmige Wolke erreichte. - Übrigens mußte man sich solche Ziele stets so wählen, $\mathrm{da} ß$ sie gelangen, andererseits durften sie auch nicht zu einfach sein, das hätte alle Spannung genommen", berichtete eine Studentin.

Derartige Vorzeichen scheinen vor allem unter Kindern und Jugendlichen allgemein zu sein, obgleich manche Informanten erwähnen, daß man darüber nie mit anderen gesprochen hat. Viele 
glauben, sie hätten diese Vorzeichen selbst erfunden. Wenn man die sich gestellte Bedingung nicht erfüllt, ist man deshalb nicht besonders deprimiert, sondern man stellt sich gleich eine neue Bedingung, die man leichter erfüllen kann. Obgleich dieser Brauch kindlich wirkt, kann doch auch ein Erwachsener in Streßsituationen ähnlich handeln oder zumindest versuchen, ob die Patience aufgeht oder nicht.

Derartige private Vorzeichen unterscheiden sich prinzipiell kaum von den sonstigen Orakeln. Mit volkstümlichen Vorzeichen sind überlieferte Bedeutungen verbunden (z. B. der Schwarzspecht fliegt neben einem plötzlich auf). Bei den oben behandelten Bedingungen und Vorzeichen will man eher das Gelingen eines Vorhabens erfahren bzw. sichern.

Oft läßt sich schwer feststellen, ob ein Vorzeichen nur als Zeichen, als Hinweis oder auch kausal, als Ursache begriffen wird. Unklar ist zum Beispiel, ob eine schwarze Katze Unglück voraussagt oder es direkt bringt, verursacht, da diese Dinge in der Regel nicht analysiert werden; das gilt eigentlich auch für die Orakel. Als Bezeichnung weist ein Vorzeichen darauf hin, daß die Natur - an sich alles, was man um sich herum beobachten kann - teilnimmt daran, was das Individuum betrifft und daß sie dem Betroffenen eine Nachricht zukommen lassen will über sein künftiges Schicksal. Die zu verschiedenen Zeiten aktualisierten Glaubensvorstellungen sind mitunter widersprüchlich. Die Vorsichtsmaßnahmen, die man z. B. nach einem bösen Vorzeichen ergreift (wie das Ausspucken nach einer schwarzen Katze), zeigen, daß man mit dem Vorzeichen auch das Ereignis zunichte macht, so daß es sich - anders als im Augenblick der Wahrnehmung - um eine Kausalbedeutung handelt.

Um überhaupt Vorzeichen befragen zu können, muß man annehmen, daß sich der Mensch mit seinen Vorstellungen als Zentrum im Universum fühlt, wo alles auf ihn einwirkt. Seine Gedanken sind bedeutungsvoll, und die ganze Natur nimmt teil 
an den Ereignissen, die ihn betreffen. Der Mensch glaubt auch, die Gedanken des anderen zu kennen. Ein anderer denkt an ihn oder spricht von ihm, wenn er selber niest, einen Gegenstand fallen läßt, wenn das Ohr rauscht, wenn sich das Schloß der Halskette vorn befindet usw. Derartige Glaubensvorstellungen zeigen einmal, welchen Glauben man der Bedeutung der Gedanken schenkt, und bringen zum andern eine willkommene Abwechslung im Alltag.

Im voraus und verfrüht

Bewundern, Prahlen und übertriebenes Loben sind uralt und weltweit bekannt als Form des Verderbens des Glücks, des Unglückbringens. Die Selbstzufriedenheit bringt ebenso Unglück wie das Loben und Preisen des anderen. Schon allein ein zufriedener Gemütszustand, ja der bloße Gedanke kann ausreichen, das Glück zu verderben; so sagt eine Informantin: „Wenn ich nur daran denke, daß ich diese oder jene Krankheit noch nicht bekommen habe, so hab' ich am nächsten Tag z. B. schon die Grippe gehabt."14 Auch in diesem Fall scheint der Gemütszustand von primärem Einfluß zu sein, die ausgesprochene Feststellung dient nur der Intensivierung oder Verstärkung.

Wenn es sich um das bedrückende Gefühl handelt, das durch den Erfolg hervorgerufen wird, so ist der Unterschied zwischen magischem Denken und sinnvollem sozialem Verhalten sehr gering. Ein Teil der Menschen gibt seinem Verhalten eine rationale Begründung: Das Leben hat sie vorsichtig gemacht, es handelt sich um Lebenserfahrung. Es ist peinlich, wenn man seine Angelegenheiten großartig anpreist und die Pläne dann doch nicht in Erfüllung gehen; außerdem soll man die anderen nicht neidisch machen. Unbemerkt verfällt man in magisches Denken, wenn durch das Glücksgefühl direkt Unglück verursacht wird oder 
wenn man der Ansicht ist, durch das Reden und Prahlen sei alles schief gegangen - ohne das wäre alles gelungen.

In diesem Zusammenhang ist es interessant zu sehen, wie ein sozial verursachter Schaden abgewehrt wird (z. B. das Loben der eigenen Angelegenheiten in Anwesenheit anderer) und wie andererseits die Zufriedenheit abgewendet wird, die man selbst spürt. Wenn andere zuhören, vermeidet man Selbstsicherheit und lobende Erwähnung der eigenen Angelegenheiten, es werden unnötige Vorbehalte in die Rede eingestreut (,ich tue das, falls ich noch lebe“, ,vielleicht im nächsten Jahr, wenn die Gesundheit es zuläßt“" usw.). Wenn man versehentlich seine Zufriedenheit zum Ausdruck gebracht hat, kann man dieses Prahlen auf zweierlei Art und Weise zurücknehmen: entweder verbal, indem man den Erfolg verneint oder indem man auf Holz klopft. Die eigenen Errungenschaften werden geringgeschätzt, die Schwierigkeiten herausgestellt (,ja, das ist schon ein gutes Auto, aber wie soll man das abbezahlen“). ${ }^{15}$ Das Klopfen auf Holz oder ähnliche Abwehrmagie gilt als häufigste magische Geste unserer Zeit.

Wenn man allein ist, klopft man nicht auf Holz, sondern man sucht seinen Gemütszustand entsprechend zu regulieren. Ein deutlich magisches Mittel ist auch, wenn man statt der Zufriedenheit eine Depression suggeriert. Man redet sich ein, daß alles schlecht steht, macht sich deshalb Sorgen, um dann wieder zu glauben, daß alles eigentlich doch in Ordnung ist. Solche Gedankengänge sind ziemlich üblich. Eine Studentin bezeichnete dies als „Theorie des Gegenteils“. In der Schule habe sie z.B. immer gedacht, sie habe eine schlechte Arbeit geschrieben, damit es in Wirklichkeit umgekehrt war.

Ein Denken im Sinne dieser „Theorie des Gegenteils“ begegnet in zahlreichen Lebenssituationen; hier einige Beispiele:

- über Autounfälle wird im Auto oft in dem Ton geredet, daß der Unfall bei entsprechender Vorsicht hätte vermieden werden können. Diesmal wird man dem Übel entgehen, weil die Gefahr im Gespräch in Worte gekleidet wurde; 
- folgendes darf man z. B. nicht tun: bei einer Einladung zu früh die Buttercreme-Torte anschneiden und sich davon nehmen, die sog. Studentenmütze eines anderen aufprobieren, während der Schwangerschaft Babykleidung stricken oder häkeln, sich im voraus auf oder über etwas Angenehmes freuen, von einer unvollendeten Arbeit reden, zu viel lachen, sich selbst dazu beglückwünschen, $\mathrm{da}$ 3 man gesund geblieben ist;

- das finnische Sprichwort „ei kaksi ilman kolmatta“ (wörtlich: „Nicht zwei ohne ein Drittes", dt. Aller guten Dinge sind drei, in Amerika sagt man: Bad things happen in threes) kann - neben anderen Bedeutungen auch Abwehrmagie enthalten: indem man das Unglück ausspricht, raubt man dem lauernden Schicksal gleichsam die böse Absicht. Das Unangenehme kann nicht geschehen oder wenn es geschieht, dann nimmt man es fatalistisch: Ich hab's ja gleich gesagt!

„Natürlich geht alles schief!“

Murphys Gesetze sind international bekannt und beliebt; in ihnen kommt das Denken, daß alles sowieso schiefgeht, anschaulich zum Ausdruck. Angeblich ist diese Sammlung 1949 im Stützpunkt Edwards in Kalifornien entstanden. Captain Ed Murphy wurde ungeduldig wegen eines Transformators, der durch einen Fehler in der Wicklung nicht funktionierte und meinte - unter Hinweis auf den betreffenden Techniker -: „Wenn man nur etwas falsch machen kann, dann macht er es falsch."

George E. Nichols, der Leiter der Sicherheits- und Qualitätskontrollen, formulierte auf dieser Grundlage das Murphysche Gesetz, das nach ein paar Monaten bereits bekannt geworden war und weiterentwickelt wurde. Folgende Behauptungen gehören z. B. zu Murphys Gesetzen:

1. Alles, was schiefgehen kann, geht schief.

2. Alles, was schiefgehen kann, geht zum schlimmsten Zeitpunkt schief.

3. Nichts ist so leicht wie es aussieht.

4. Wenn mehrere Dinge schiefgehen können, dann geht das schief, was am meisten Schaden anrichtet.

5. Die Natur ist stets auf der Seite des geheimen Fehlers. 
Die Beliebtheit der Murphy-Gesetze hat man mit ihrem parodistischen Charakter erklärt: Sie verspotten die amerikanische Ordnungsmanie, das Streben nach Wissenschaftlichkeit und den bewunderten Optimismus. ${ }^{16}$ In einer Hinsicht berühren sie den latenten Aberglauben im Menschen. Die Äußerungen wecken ein geheimnisvolles Gefühl der Befriedigung, da man das Schicksal hat überlisten können, indem man dessen böse Absichten im voraus aufdeckte.

Nach dieser „Überlistungstheorie“ versucht der Mensch das Schicksal oder die Naturgesetze irrezuführen, indem er zeigt, daß er nichts mehr erwartet. Nun kann das Positive, was man erhofft und erwartet hat, in Ruhe eintreten. Durch das Vortäuschen einer bestimmten Einstellung erreicht man schließlich doch das Gewünschte. Es gibt $\mathrm{z}$. B. folgende Überlistungssituationen:

- wenn man sich eine Zigarette anzündet, während man auf die Straßenbahn wartet, kommt sie bestimmt (das gilt auch für das bestellte Essen im Restaurant),

- wenn man will, daß das Telefon klingelt, muß man in die Badewanne oder unter die Dusche gehen,

- wenn ein Anhalter sich in die Büsche verzieht, kommt das ersehnte Auto sofort,

- man nimmt den Regenschirm mit, so wird es nicht regnen,

- wenn man sich einredet, daß man beim Angeln nichts fängt, beißt der Fisch sofort an,

- wenn man eine Versicherung hat, passiert nichts Schlimmes.

„Ich fürchtete, meinen Hunden würde etwas passieren, wenn ich nicht bereit wäre, die Versicherungsgebühren zu bezahlen“, meinte eine Studentin. Mit Hilfe der Versicherung stellt man sich auf ein Unglück ein und nimmt so dem Schicksal im voraus dessen böse Absicht und zahlt für sein Glück mit Geld.

Wir haben oben halb unbewußte Glaubensvorstellungen erwähnt, die dennoch in der inneren Gedankenwelt wirken. Viele Menschen sind der Ansicht, daß es auf der Welt ebenso viel Glück wie Unglück gibt; wenn man lange und reichlich Glück 
hat, muß man sich auf Unglück einstellen. Eine junge Frau erklärt ihre Fehler, die sie am Arbeitsplatz machte, wie folgt: ,,Seit es mit meinem Freund vorbei ist, habe ich nur Unglück. Nichts gelingt, was ich auch anfange. Ich habe jetzt ganz deutlich eine Pechsträhne, da kann man nichts machen."

Eine „Pechsträhne“ kann man auch in der Phantasie erleben und sie damit im realen Leben verhindern. Der Mensch glaubt, seine Sorge habe einen Nutzen, wie Thomas Mann es in seinem Roman „Der junge Joseph“ so anschaulich schildert. Als Jakob die Nachricht von Josephs Tod erhält, empfindet er das Unglück als ungerecht und fragt, ob er nicht durch seine Furcht und Sorge das Böse abgewendet habe und dem Schicksal im vorhinein die bösen Gedanken geraubt habe.

$\mathrm{Zu}$ den unausgesprochenen Vorstellungen gehört auch der Gedanke, daß der Mensch als Gegenspieler eine Art lautlosen Mitläufer habe, eine Kraft, die das Übernatürliche repräsentiert, wozu er jedoch keine persönliche Beziehung besitzt. Diese Kraft reagiert auf die Freude und Zufriedenheit des Menschen, indem sie ihm das voreilige Glück raubt.

Schicksal und Naturgesetze

Die oben geschilderten Gedankenmuster - die „Theorie des Gegenteils" und das Überlisten des Schicksals in seinen verschiedenen Formen - sind den meisten Menschen in der heutigen Kultur bekannt. Wenn man magisches Wissen sammelt, kommen derartige Dinge nicht zum Vorschein, falls man nicht deutlich und detailliert danach fragt. Einerseits betrachtet sich der Mensch gleichsam von außen her und amüsiert sich über seine Vorstellungen und berichtet lachend über seine magischen Sitten, wenn es aber ernst wird, dann hält er daran fest. 
Obwohl die Überlieferung eine soziale Erscheinung ist, aktualisiert sich ein Teil davon nur und ausschließlich in den Gedanken des Menschen. In Gedanken flucht der Mensch auch und verwendet dann oft andere, derbere Ausdrücke, als wenn er laut etwas verwünscht. In Gedanken erinnert er sich an den Text eines Volksliedes oder einer Erzählung, in Gedanken erzählt er sie seinen vermeintlichen Zuhörern.

Viele magische Gedankenmuster setzen einen vorgestellten Gegenspieler voraus, der einen beobachtet, der einem zuhört, der einen kommentiert. Die Gestalt dieses Wesens oder dieser Kraft wird von den Interviewten nicht ohne weiteres präzisiert, offenbar haben sie auch kein genaueres Bild davon. Worin besteht die Gefahr, die man heutzutage fürchtet?

Im älteren finnland-schwedischen Material werden drei Möglichkeiten als Thema dieser Bedrohung genannt: 1. Die „Rache des Schicksals“ führt zu einer Veränderung, 2. Gott nimmt einem das, womit man prahlt, 3. Angeberei weckt den Neid und die Böswilligkeit des Nachbarn. ${ }^{17}$ Die Gedanken des modernen Menschen konzentrieren sich vielleicht unbestimmt auf die Rache des Schicksals, falls man über das Ganze nachdenkt. Möglich ist auch, daß neuartige Vorstellungen über Strahlungen im Weltall entstehen. Solche unbestimmten Strahlungstheorien sind in den vergangenen Jahren zum Beispiel zur Untermauerung des astrologischen Weltbildes vorgebracht worden.

Als volkstümliches Wissen enthält die Magie Ratschläge, wie man das Glück erreicht und das Unglück vermeidet. Das Glück ist ein zentraler Begriff, und die diesbezüglichen Glaubensvorstellungen sind im Bereich zwischen dem bewußten und dem unbewußten Denken angesiedelt. Auf direkte Fragen über die diversen Wechselwirkungen erhält man leicht fingierte resp. unechte Antworten, weil der Befragte nie bewußt analysiert, wie $\mathrm{x}$ auf $\mathrm{y}$ wirkt. Der schwedische Überlieferungsforscher $\mathrm{C}$. $\mathrm{W}$. von Sydow hat bereits in den dreißiger Jahren festgestellt, daß es 
ein ebenso großer wie üblicher Irrtum unter den Wissenschaftlern ist, anzunehmen, das Volk denke, irgendetwas sei die Grundlage für etwas anderes; viel häufiger, vermutlich meistens, wird lediglich festgestellt, $\mathrm{da} ß$ eine Erscheinung die Ursache für eine andere ist. 18

Zur Bedeutung der Stimmungsmagie

Mitunter beeinflußt man das Schicksal, indem man die Stimmung reguliert: man bagatellisiert das Glück und bildet sich das Unglück ein (z. B. in der Schule nach einer Prüfung oder Arbeit), in anderen Situationen sucht man eine ruhige, ausgeglichene Stimmung (Fischereizauber). Im Sinne der letztgenannten Beeinflussung wollen auch Schüler oder Studenten z. B. „brav" sein vor einer entscheidenden Prüfung, asketisch leben oder anderweitig sich um das Glück verdient machen.

In bestimmten, vom Glück abhängigen Bereichen, wie z. B. dem Kartenspiel, gibt es Vorstellungen davon, wie die Stimmung auf das Glück Einfluß nimmt. Dostojewski berichtet in seiner autobiographischen Schilderung, wie der Spieler glaubt, er gewinne, wenn er sich beim Spielen nicht erregt. Entsprechende Anweisungen werden in einer Broschüre für Besucher von Spielcasinos gegeben: Man solle eine das Gewinnen fördernde Stimmung haben, zuversichtlich, ruhig und fast scherzhaft aufgelegt sein.

Alles menschliche Handeln wird von den Gefühlen beeinflußt, angefangen von der Erinnerung. Schon im Alltag kann man die Feststellung machen, daß eine Arbeit, die man ruhig und ausgeglichen angeht, besser gelingt, als wenn man nervös ist. Die sog. ESP-Versuche untermauern die Auffassung, daß eine gute Stimmung die Voraussetzung ist für gutes Glück z. B. beim Kartenspiel. Versichert man einer Person, daß sie Glück hat, dann hilft 
ihr diese Versicherung tatsächlich. Tests haben ergeben, daß erfolgreiche Geschäftsleute mehr ESP-Fähigkeiten haben als andere Menschen. ${ }^{19}$ Offenbar kennen wir längs nicht alle Wirkungen des Gefühlslebens, doch ist eine harmonische und entspannte Stimmung aus vielen Gründen etwas Erstrebenswertes.

Nun gibt es auch die suggerierte Depression, mit der der Betroffene schließlich zufrieden ist. Es handelt sich dabei auch um Stimmungen, die durch den Glauben entstehen, daß alles sowieso schiefgeht. Auf diese Weise soll das erschütterte Gleichgewicht wiederhergestellt werden, das hervorgerufen wird durch die Furcht vor Unglïck oder durch eine unbestimmte Bedrängnis.

Helfen nun die magischen Vorkehrungen dem Menschen zu einem fröhlichen Optimismus, wenn man die Angelegenheit vom Standpunkt der Mentalhygiene betrachtet? In der Regel führen optimistische Gedanken zu positiven Ergebnissen und zum Gelingen, pessimistische dagegen zu negativen Ergebnissen und zum Mißlingen. Eine positive, aktive Lebenseinstellung galt vor allem in den Vereinigten Staaten lange als einer der Schlüsselbegriffe für die Entstehung von Unternehmungsgeist. Danach kann man seine Leistungen verbessern, wenn man sich eine ständig positive Einstellung zu den Dingen zueigen macht; man muß sich das Ziel deutlich, lebendig und detailliert vorstellen.

Obgleich magische Glaubensvorstellungen in einer bedrängenden Situation Streß verursachen können, deprimieren sie doch auf längere Sicht weiter und verhindern die Wahrnehmung realer Möglichkeiten, durch die positive Veränderungen im Leben möglich wären. Die Untersuchung des magischen Denkens kann für den Überlieferungsforscher wichtige Gebiete der angewandten Forschung eröffnen. 
Widersprüchliche Glaubensvorstellungen

Das Streben, durch seine Gedanken Einfluß zu nehmen auf ein gerade geschehendes Ereignis, ist eine allgemeine Form der Magie und tritt bei Menschen auf, die - danach befragt - die Möglichkeit einer solchen Beeinflussung nicht zugestehen würden. Die Realität sieht jedoch $\mathrm{zu}$ verschiedenen Zeitpunkten unterschiedlich aus, und im Alltagsleben werden jeweils die Grundvermutungen aktualisiert, die in dem Augenblick zweckmäßig erscheinen.

Der moderne Mensch betrachtet sich vielleicht als Christen, glaubt jedoch gleichzeitig an die Wiedergeburt, beschäftigt sich mit Horoskopen und spuckt bei Bedarf aus, wenn er eine schwarze Katze sieht. Paul Veyne, der den Glauben der Griechen an die Mythen untersucht hat, schreibt darüber, wie er fast neurotische Angst vor Gespenstern hat, wie er z. B. fürchtet, daß ihm sein verstorbener Freund erscheint, obwohl er gleichzeitig eine bloße Fiktion ohne jegliche Beweiskraft in dieser Vorstellung sieht. Er kommt zu dem einfachen Schluß, daß wir die verschiedenen Wahrheiten nicht mit dem gleichen Teil unseres Kopfes denken. ${ }^{20}$

Zahlreiche Widersprüche im alten Volksglauben haben sich ganz offensichtlich deshalb lebendig erhalten, weil sie nie gleichzeitig aktualisiert werden. Der Mensch hat sein Weltbild nicht zu einem logischen System geschliffen; die aus der Kindheit überkommenen Vorstellungen zum Beispiel hat er dem Gesamtbild nicht angeglichen. Er ist auch als Erwachsener nicht imstande, eine Spinne zu töten, obwohl er andererseits in dem Verbot keinen Sinn sieht.

Der eingangs zitierte Hinweis von Stevenson, daß der Mensch in der Kammer seines Gehirns und nicht in der äußeren Wirklichkeit unter Salzen und Säuren lebt, erweist sich im Lichte dieser Vorstellungen als wahr. In der inneren Kammer erfährt die Wirklichkeit eine starke Bearbeitung durch das menschliche Ich: Das Weltall prophezeit dem Menschen sein Schicksal, wenn er 
bis hundert zählt, geschehen bestimmte Dinge, abhängig von seinem Glauben oder Unglauben kommt es zu Ereignissen, seine Fröhlichkeit oder Traurigkeit hat Auswirkungen, ein Gegenspieler tritt auf, jemand/etwas will sich mit ihm messen. Zu keinem Augenblick ist er unbedeutend. Man könnte dieses Denken als pseudoreligiös bezeichnen, obwohl hier das Verhältnis zu einem persönlichen Gott fehlt.

Hat die Stimmungsmagie einen Platz im Weltbild des Menschen? Man kann diesen Bereich natürlich leicht als Unterschicht abtun, die nicht ,aufgeräumt" ist, nicht mit den oberen Schichten zusammenpaßt. Zum Weltbild gehören aber auch die undifferenzierten Empfindungen, die Grundstimmungen, durch die der Mensch empirisch mit der Welt in Verbindung tritt. Angst, Aggression und Bedrängnis sind ebenfalls auf die Welt gerichtete Bedeutungsbeziehungen, auch wenn sie nicht klar gegliedert sind. Das Weltbild ist jedoch von derartigen Gefühlen sehr stark abhängig. Was Immanuel Kant im 18. Jahrhundert gesagt hat, dem kann sich auch der Psychiater von heute anschließen: „Auf der großen Karte unseres Geistes gibt es nur wenige erleuchtete Stellen... das Gebiet der dunklen Vorstellungen ist am größten“ (Anthropologie 1789).

\section{Anmerkungen}

1. Dr. Jekyll and Mr. Hyde (1886).

2. Malinowski, Bronislaw 1960: Magia, tiede ja uskonto (Porvoo), S. 16-17 (Engl. Magic, Science and Religion and Other Essays).

3. Rauhala, Lauri 1984: Tiedostamaton fenomenologisessa tajunnan analyysissa. In: Tietoisuus ja alitajunta (Porvoo-Helsinki-Juva).

4. Inha, I. K. 1911: Kalevalan laulumailta (Helsinki), S. 69.

5. Genauer behandelt wird dieses Material in der Arbeit „Nykymagian käsikirja“" (Helsinki 1988).

6. Vuorela, Toivo 1967: Der böse Blick im Lichte der finnischen Überlieferung. In: FF Communications 201 (Helsinki), S. 14.

7. Vuorela, op. cit., S. 179-186. 
8. Virtanen, Leea 1976: „Kateus vie kalat vedestä“. In: Kalevalaseuran vuosikirja (Porvoo-Helsinki), S. 250-266.

9. Konkka, Unelma 1985: Ikuinen ikävä (Mänttä), S. 179-185.

10. Nilsson, Martin P. 1950: Griechischer Glaube (Bern), S. 62-71.

11. „Piaget has certainly demonstrated that children pass through stages of magical and animistic thinking and research in different cultures shows that this is likely to be a universal process“ (Jahoda, Gustav 1970: The Psychology of Superstition (Pelican books), S. 107.

12. Eino Kaila 1934: Persoonallisuus (Helsinki), S. 150.

13. Achté, Kalle - Alanen, Yrjö O. - Tienari, Pekka 1973: Psykiatria (Porvoo), S. 210-214.

14. Leea Virtanen 1976, Lagom lycka är bäst. In: Nordisk folktro, Fataburen (Lund), S. 257.

15. Virtanen, op. cit., S. 260.

16. Dundes, Alan - Pagter, Carl R. 1975: Work Hard and You Shall Be Rewarded (Indiana University Press), S. 69.

17. Finlands Svenska Folkdiktning VII 3, 1952 (København), S. 105.

18. Sydow, C. W. 1971: Det ovanliga i tro och sed. In: Folkdikt och folktro, utgiven av Anna Birgitta Rooth, S. 205-206.

19. Rao, K. R. 1974: Psi and Personality. In: New Directions in Parapsychology, edited by John Beloff (London).

20. Veyne, Paul 1988: Did the Greeks Believe in Their Myths? An Essay on the Constitutive Imagination (Chicago and London), S. 87. 